\title{
Drug Induced Ototoxicity
}

\section{Ryan W and Sachin D*}

LECOM-School of Pharmacy Bradenton, FL, USA

Drugs causing damage to the inner ear are commonly referred as ototoxic and can result in symptoms of tinnitus, vertigo or hearing loss. Specifically, if hearing is impaired they are designated as "cochleotoxic" and if balance is impaired, they are termed "vestibulotoxic" [1]. Ototoxicity remains a clinical concern due to its reported association with at least 130 medications [2]. Symptom severity is varied among patients, but in general, initially includes high-frequency hearing loss. Unfortunately, the incidence of otoxicity has been largely underreported because high-frequency hearing loss does not tend to interfere with routine communication and usually goes unnoticed. Perhaps the most disconcerting issue with cochleotoxic drugs is if hearing impairment becomes permanent. This can significantly impact quality of life, and therefore should be considered when choosing drug therapy to minimize risk. Thankfully, many reported ototoxic medications such as the aminoglycoside antibiotics have well documented risk or are considered alternative therapy. However, with renewed interest in the use of prophylactic antibiotics to improve quality of life and reduce frequency of exacerbations in chronic obstructive pulmonary disease (COPD) it is timely to discuss this potentially irreversible complication.

Antibiotics and antineoplastic agents are perhaps the most commonly used medications that can cause hearing loss. Although both cause ototoxicity due to oxidative stress, the mode of cell death differs. Cisplatin primarily triggers an apoptotic cell death pathway, whereas aminoglycosides trigger both necrotic and apoptotic cell death pathways. Understanding of this mechanism of action has aided the development of novel ways to protect the cochlea [1]. Aminoglycosides have a long-standing history and all oral formulations are considered to have some form of ototoxicity. While the overall risk of ototoxicity associated with any topical aminoglycoside remains low, the overall prevalence of complications such as acute otitis media warrants caution with routine use should another agent with similar efficacy be available [3]. It should be noted that the clinical impact of ototoxicity due to aminoglycosides is minimal in current practice with appropriate monitoring. This is not the case however with the use of certain macrolide antibiotics such as azithromycin. While periodic use does not impose a significant threat, chronic administration can cause permanent hearing loss $[4,5]$. This is of concern with renewed interest in the use of prophylactic antibiotic use for the treatment of COPD. A recent review assessing the impact of azithromycin, erythromycin, clarithromycin and moxifloxacin found that continuous use resulted in a clinically significant benefit in reducing exacerbations in COPD patients [4]. At first glance, these are promising results in a disease that is difficult to manage, however among other adverse events, hearing loss was commonly reported [4]. As a result, the general recommendation is to avoid antibiotic use, other than for treating infectious exacerbations of COPD and other bacterial infections $[5,6]$.

Beyond recognition of commonly used medications that can cause ototoxicity, clinicians can consider several factors when designing a medication regimen including, but not limited to concomitant use of ototoxic agents, dose and frequency of administration and renal function. Not surprisingly, co-administration of ototoxic agents, utilization of high dosages, or frequent administration may warrant close assessment through monitoring of laboratory values or clinical status of the patient. This is especially important in the case of renal decomposition, as this may increase the risk for ototoxicity. Antioxidants, including salicylates, have been shown to have otoprotective effects when used with aminoglycosides [7]. However, this approach to prevention of toxicity should be used with caution as it could be associated with additional adverse effects and drug interaction potential.

Ototoxicity is a detrimental adverse effect, but its impact can be minimized by following precautionary standards. Assessment of any past medical history including hearing loss, dizziness or tinnitus, baseline hearing and review of the medication regimen for ototoxic agents help reduce overall incidence. Additionally, utilization of ototoxic agents at the lowest effective dose for the shortest duration necessary should be emphasized. Further research is needed to prevent or augment ototoxicity, but utilization of these techniques can help minimize its impact in practice.

\section{References}

1. Schacht J, Talaska AE, Rybak LP (2012) Cisplatin and aminoglycoside antibiotics: hearing loss and its prevention. Anat Rec295: 1837-1850.

2. Seligmann H, Podoshin L, Ben David J, Fradis Goldser M (1996) Drug-induced tinnitus and other hearing disorders.Drug Saf 14: 198-212.

3. Roland PS, Stweart MG, Hannley M, Friedman R, Manolidis S, et al. (2004) Consensus panel on role of potentially ototoxic antibiotics for topical middle ear use: Introduction, methodology and recommendations. Otolaryngol Head Neck Surg. 130: S51-6

4. Herath SC, Poole P (2013) Prophylactic antibiotic therapy for chronic obstructive pulmonary disease (COPD). Cochrane Database of Syst Rev.

5. Herath SC, Poole P (2013) Prophylactic antibiotic therapy for chronic obstructive pulmonary disease (COPD). Cochrane Database of Systematic Reviews, Issue 11. Art. No.: CD009764.

6. Global Initiative for Chronic Obstructive Lung Disease (GOLD). (2014) Global strategy for the diagnosis, management, and prevention of chronic obstructive pulmonary disease. Vancouver (WA): Global Initiative for Chronic Obstructive Lung Disease (GOLD)

7. Rybak LP, Ramkumar V (2007) Ototoxicity. Kidney Int 72: 931-935.

*Corresponding author: Sachin D, LECOM-School of Pharmacy, Bradenton FL, 34203, USA, Tel: 941-782-5941; E-mail: sdevi@lecom.edu

Received October 16, 2014; Accepted October 17, 2014; Published October 20 2014

Citation: Ryan W, Sachin D (2014) Drug Induced Ototoxicity. Clin Exp Pharmaco 4: e132. doi:10.4172/2161-1459.1000e132

Copyright: $\odot 2014$ Ryan W, et al. This is an open-access article distributed under the terms of the Creative Commons Attribution License, which permits unrestricted use, distribution, and reproduction in any medium, provided the original author and source are credited. 